\title{
Generalized Space Time Block Coded Spatial Modulation Systems
}

\author{
Lixia Xiao ${ }^{a}$, Pei Xiao ${ }^{a}, \mathrm{Chao} \mathrm{Xu}^{b}$, Ibrahim A. Hemadeh ${ }^{a}$, De $\mathrm{Mi}^{a}$, Wanming Hao ${ }^{a}$ \\ ${ }^{a} 5 \mathrm{G}$ Innovation Centre, Institute for Communication Systems, University of Surrey, United Kingdom, \\ ${ }^{b}$ School of Electronics and Computer Science, University of Southampton \\ (e-mail: xiaolixia_cool@163.com, p.xiao@surrey.ac.uk)
}

\begin{abstract}
In this paper, Generalized Space-Time Block Coded Spatial Modulation (GSTBC-SM) is proposed for Multiple-Input and Multiple-Output (MIMO) system, which can be extended into an arbitrary even number of Transmit Antennas (TAs). The proposed GSTBC-SM scheme employs the hybrid concepts of Generalized Space-Time Block Coding (GSTBC) and Spatial Modulation (SM) to further exploit the diversity benefits of GSTBC using sparse Radio Frequency (RF) chains. To be more specific, the information bits are divided into $N_{u}$ groups and each group is modulated by SM scheme. Finally, the $N_{u}$ symbols are invoked for GSTBC structure. In order to demonstrated the advantages of our proposed GSTBCSM schemes, the theoretical Average Bit Error Probability (ABEP) of our proposed GSTBC-SM is derived. Both our analytical and simulation results demonstrated that the proposed GSTBC-SM scheme is capable of providing considerable performance gains over the corresponding GSTBC schemes at the same transmit rate associated with the same number of $\mathrm{RF}$ chains.
\end{abstract}

Index Terms - Spatial Modulation (SM), Space Time Block Coding (STBC), Diversity gain.

\section{INTRODUCTION}

$\mathbf{S}$ PACE Time Block Coding (STBC) [1]-[2] is a classic Multiple Input Multiple Output MIMO technique, which is capable of obtaining high diversity gain by transmitting same data information via different time slots. There are two classic categories of conventional STBC schemes: Orthogonal STBC (OSTBC) [1]- [4] and Quasi-OSTBC schemes [5]- [11]. To be more specific, in [1], the author Alamouti first proposed the OSTBC scheme with $N_{t}=2$ Transmit Antennas (TAs). Then, the authors of [2]- [4] further extended orthogonal STBC concept into other number of TAs. More importantly, the authors of [4] presented a large OSTBC scheme with $N_{t}=18$. Meanwhile, the Quasi-OSTBC schemes with a small number of TAs were developed in [5]- [11]. Due to its complexity of code design and high cost of Radio Frequency (RF) chains, conventional STBC schemes are unsuitable for massive MIMO systems.

In order to further exploit the diversity gain of massive MIMO relying on sparse RF chains at the transmitter, STBC based Spatial Modulation (SM) scheme [12]- [18] were developed in [19]- [23]. In the STBC-SM schemes, the information bits were conveyed by the indices of activated Transmit Antenna Combination (TAC) as well as Amplitude Phase Modulation (APM) symbols. To be more specific, the STBC-SM scheme with two RF chains was firstly developed in [19]. Then, the high transmit rate and Modified Codeword based variants were developed in [20]- [23]. However, the TAC design of these STBC-SM schemes was based on different amplitudes and phases, which became very complex for the large number of TAs. More importantly, the diversity gains of these schemes decreased as the value of $N_{t}$ increases. In order to address this issue, a novel STBC-SM scheme, which we called Alamouti-SM scheme was proposed in [24]. In the Alamouti-SM scheme, the SM symbols were taken as a whole vector to involve in the Alamouti coding. Hence, the TAC design became easy for an arbitrary number of TAs. More importantly, the diversity gains kept constant as $N_{t}$ increases. However, the STBC-SM schemes of [19]- [24] were mainly designed for $N_{u}=2$, where $N_{u}$ is the number of activated TAs.

In order to exploit the diversity gain of STBC-SM with large number of $N_{u}$, the double space-time transmit diversity based spatially modulated orthogonal space-time block codes scheme relying on four RF chains at the transmitter was developed in [25]. However, the total number of space codewords was $4^{N_{t}-2}$, which is very large at massive MIMO, result in high complexity of space codewords design. To the best of authors' knowledge, the generalize STBC-SM scheme with low-complexity has not been designed yet.

Against the above background, the contributions of this paper are summarized as follows:

1) A generalized STBC-SM (GSTBC-SM) scheme is proposed in this paper. In the proposed GSTBCSM scheme, the information bits are divided into $N_{u}$ groups and SM is employed for each group. Then the $N_{u}$ SM symbols are invoked into conventional STBC structure. Hence, the proposed GSTBC-SM is capable of maintaining both benefits of STBC and SM schemes.

2) Furthermore, the orthogonality and Average Bit Error Probability (ABEP) are derived for our proposed GSTBC-SM scheme, which are confirmed by our simulation results.

3) Both theoretical and simulation results are demonstrated that the proposed GSTBC-SM schemes are capable of providing significant performance gain over the conventional STBC and STBC-SM schemes. 
The remainder of this paper is organized as follows. Section II gives a rudimentary introduction to the conventional STBC systems. Section III introduces the system model of the proposed GSTBC-SM system. Section IV analyzes the orthogonality and the upper bound of the ABEP. Section V presents our simulation results. Finally, Section VI concludes this paper.

Notation: $\|\cdot\|_{2}$ denotes the two norm of a matrix; | | represents the magnitude of a complex quantity; $(\cdot)^{*}$, $(\cdot)^{T}$ and $(\cdot)^{H}$ stand for the conjugate, transpose and the Hermitian transpose of a vector/matrix, respectively.

\section{AN OVER REVIEW OF EXISTING STBC SCHEMES}

In this section, conventional STBC schemes are introduced. For simplicity, we mainly introduce the structures of Alamouti, Orthogonal STBC schemes.

\section{A. Alamouti Scheme}

For Alamouti scheme, the information bits are divided into two groups, which are modulated into two $M$-APM symbols $x_{1}$ and $x_{2}$. Then the transmit signal are obtained by Alamouti coding as

$$
G_{22}=\left[\begin{array}{cc}
x_{1} & -x_{2}^{*} \\
x_{2} & x_{1}^{*}
\end{array}\right]_{2 \times 2}
$$

According to (1), we have $G_{22} G_{22}^{H}=2 \mathbf{I}_{2}$.

\section{B. Orthogonal STBC scheme with $R=3 / 4$}

For OSTBC scheme with $R=3 / 4$, the information bits are divided into 3 groups, which are modulated into three $M$-APM symbols as $x_{1}, x_{2}$ and $x_{3}$. Then the transmit signal are obtained by STBC coding as

$$
G_{434}=\left[\begin{array}{cccc}
x_{1} & -x_{2}^{*} & x_{3}^{*} & 0 \\
x_{2} & x_{1}^{*} & 0 & x_{3}^{*} \\
x_{3} & 0 & -x_{1}^{*} & -x_{2}^{*} \\
0 & x_{3} & x_{2} & -x_{1}
\end{array}\right]_{4 \times 4}
$$

and we have $G_{434} G_{434}^{H}=3 \mathbf{I}_{4}$;

\section{Orthogonal STBC scheme with $R=1 / 2$}

For OSTBC scheme with $R=1 / 2$, the information bits are divided into 4 groups, which are modulated into four $M$-APM symbols as $x_{1}, x_{2}, x_{3}$ and $x_{4}$. Then the transmit signal are obtained by STBC coding as

$$
G_{448}=\left[\begin{array}{cccccccc}
x_{1}-x_{2} & -x_{3} & -x_{4} & x_{1}^{*} & -x_{2}^{*} & -x_{3}^{*} & -x_{4}^{*} \\
x_{2} & x_{1} & x_{4} & -x_{3} & x_{2}^{*} & x_{1}^{*} & x_{4}^{*} & -x_{3}^{*} \\
x_{3}-x_{4} & x_{1} & x_{2} & x_{3}^{*} & -x_{4}^{*} & x_{1}^{*} & x_{2}^{*} \\
x_{4} & x_{3} & -x_{2} & x_{1} & x_{4}^{*} & x_{3}^{*} & -x_{2}^{*} & x_{1}^{*}
\end{array}\right]_{4 \times 8},
$$

and we have $G_{448} G_{448}^{H}=8 \mathbf{I}_{4}$.

Furthermore, other generalized STBC schemes can be found in [2]- [11].

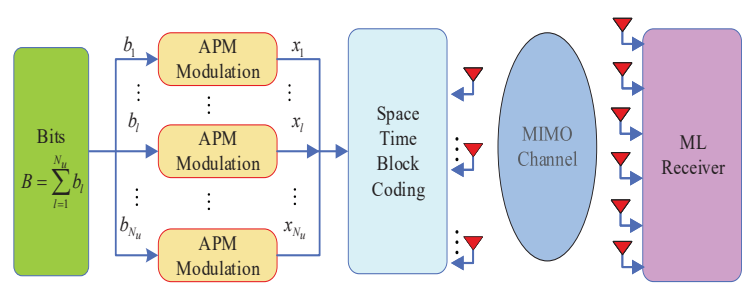

(a) Conventional GSTBC system

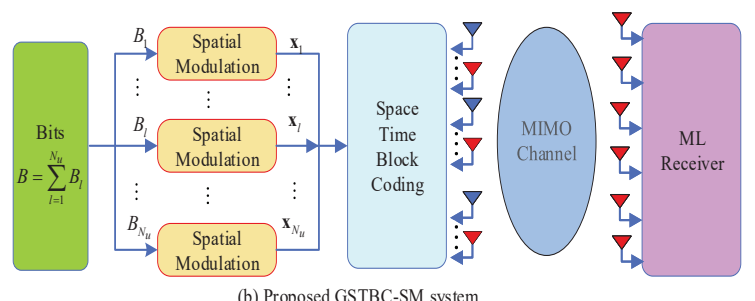

Fig. 1. System models of the conventional GSTBC and the proposed GSTBC-SM schemes.

\section{Proposed Generalized Space Time Block Coding Spatial Modulation system}

In this section, a GSTBC-SM structure is proposed for an arbitrary even number of TAs, which is shown in Fig. 1. To be more specific, the information bits are divided into $N_{u}$ gropus based on the value of $N_{t}$ and STBC structure. For the given STBC structure and value of $N_{u}$, the value of $N_{t}$ will also be divided into $N_{u}$ values as $n_{l} l=1, \ldots, N_{u}$. If $n_{l}$ is a power of 2 , we will get the $l$-th SM symbol $\mathbf{x}_{l}$ by SM mapping of [12], otherwise, $\mathbf{x}_{l}$ is obtained by the mapping method of [24]. Next, the $N_{u}$ SM symbols will be invoked into STBC structure, where the conventional constellation points are replaced by the SM symbols. We will introduced our proposed GSTBC-SM structure by specific STBC schemes.

\section{A. Proposed OSTBC-SM with $R=3 / 4$}

For OSTBC-SM scheme with $R=3 / 4$, the information bits are divided into 3 groups, which are modulated into three $\mathrm{SM}$ symbols as $\mathbf{x}_{1}, \mathbf{x}_{2}$ and $\mathbf{x}_{3}$. Then the transmit signal are obtained by STBC coding as

$$
\mathbf{S}_{434}=\left[\begin{array}{cccc}
\mathbf{x}_{1} & -\mathbf{x}_{2}^{*} & \mathbf{x}_{3}^{*} & 0 \\
\mathbf{x}_{2} & \mathbf{x}_{1}^{*} & 0 & \mathbf{x}_{3}^{*} \\
\mathbf{x}_{3} & 0 & -\mathbf{x}_{1}^{*} & -\mathbf{x}_{2}^{*} \\
0 & \mathbf{x}_{3} & \mathbf{x}_{2} & -\mathbf{x}_{1}
\end{array}\right]_{N_{t} \times 4}
$$

Example: Assuming that $N_{t}=8$ and

$$
\mathbf{x}_{1}=\left[\begin{array}{c}
0 \\
s_{1}
\end{array}\right], \mathbf{x}_{2}=\left[\begin{array}{c}
s_{2} \\
0
\end{array}\right], \mathbf{x}_{3}=\left[\begin{array}{c}
0 \\
s_{3}
\end{array}\right]
$$


we have

$$
\mathbf{S}_{434}=\left[\begin{array}{cccc}
0 & -s_{2}^{*} & 0 & 0 \\
s_{1} & 0 & s_{3}^{*} & 0 \\
s_{2} & 0 & 0 & 0 \\
0 & s_{1}^{*} & 0 & s_{3}^{*} \\
0 & 0 & 0 & -s_{2}^{*} \\
s_{3} & 0 & -s_{1}^{*} & 0 \\
0 & 0 & 0 & 0 \\
0 & s_{3} & s_{2} & -s_{1}
\end{array}\right]_{8 \times 4}
$$

\section{B. Proposed OSTBC-SM scheme with $R=1 / 2$}

For OSTBC-SM scheme with $R=1 / 2$, the information bits are divided into 4 groups, which are modulated into four SM symbols as $\mathbf{x}_{1}, \mathbf{x}_{2}, \mathbf{x}_{3}$ and $\mathbf{x}_{4}$. Then the transmit signal are obtained by STBC coding as

$$
\mathbf{S}_{448}=\left[\begin{array}{cccccccc}
\mathbf{x}_{1} & -\mathbf{x}_{2} & -\mathbf{x}_{3} & -\mathbf{x}_{4} & \mathbf{x}_{1}^{*} & -\mathbf{x}_{2}^{*} & -\mathbf{x}_{3}^{*} & -\mathbf{x}_{4}^{*} \\
\mathbf{x}_{2} & \mathbf{x}_{1} & \mathbf{x}_{4} & -\mathbf{x}_{3} & \mathbf{x}_{2}^{*} & \mathbf{x}_{1}^{*} & \mathbf{x}_{4}^{*} & -\mathbf{x}_{3}^{*} \\
\mathbf{x}_{3} & -\mathbf{x}_{4} & \mathbf{x}_{1} & \mathbf{x}_{2} & \mathbf{x}_{3}^{*} & -\mathbf{x}_{4}^{*} & \mathbf{x}_{1}^{*} & \mathbf{x}_{2}^{*} \\
\mathbf{x}_{4} & \mathbf{x}_{3} & -\mathbf{x}_{2} & \mathbf{x}_{1} & \mathbf{x}_{4}^{*} & \mathbf{x}_{3}^{*} & -\mathbf{x}_{2}^{*} & \mathbf{x}_{1}^{*}
\end{array}\right]_{N_{t} \times 8} .
$$

Example: Assuming that $N_{t}=8$ and

$$
\mathbf{x}_{1}=\left[\begin{array}{c}
0 \\
s_{1}
\end{array}\right], \mathbf{x}_{2}=\left[\begin{array}{c}
s_{2} \\
0
\end{array}\right], \mathbf{x}_{3}=\left[\begin{array}{c}
0 \\
s_{3}
\end{array}\right], \mathbf{x}_{4}=\left[\begin{array}{c}
s_{4} \\
0
\end{array}\right],
$$

the transmit signal can be expressed as

$$
\mathbf{S}_{448}=\left[\begin{array}{cccccccc}
0 & -s_{2} & 0 & -s_{4} & 0 & -s_{2}^{*} & 0 & -s_{4}^{*} \\
s_{1} & 0 & -s_{3} & 0 & s_{1}^{*} & 0 & -s_{3}^{*} & 0 \\
s_{2} & 0 & s_{4} & 0 & s_{2}^{*} & 0 & s_{4}^{*} & 0 \\
0 & s_{1} & 0 & -s_{3} & 0 & s_{1}^{*} & 0 & -s_{3}^{*} \\
0 & -s_{4} & 0 & s_{2} & 0 & -s_{4}^{*} & 0 & s_{2}^{*} \\
s_{3} & 0 & s_{1} & 0 & s_{3}^{*} & 0 & s_{1}^{*} & 0 \\
s_{4} & 0 & -s_{2} & 0 & s_{4}^{*} & 0 & -s_{2}^{*} & 0 \\
0 & s_{3} & 0 & s_{1} & 0 & s_{3}^{*} & 0 & s_{1}^{*}
\end{array}\right]_{8 \times 8}
$$

Let $\mathbf{H} \in \mathbb{C}^{N_{r} \times N_{t}}$ and $\mathbf{N} \in \mathbb{C}^{N_{r} \times T}$ denote the MIMO channel matrix and the noise matrix with $T$ time slots, whose elements obey the complex Gaussian distributions associated with $\mathcal{C N}(0,1)$ and $\mathcal{C N}\left(0, \sigma^{2}\right)$, respectively, where $\sigma^{2}$ is the noise variance. According to (4)-(7), the received signal matrix $\mathbf{Y}_{t} \in \mathbb{C}^{N_{r} \times T}$ consisting of a pair of time slots can be expressed as

$$
\mathbf{Y}=\mathbf{H S}+\mathbf{N}
$$

Hence, the optimal Maximum Likely-hood (ML)-based demodulator can be formulated as

$$
\hat{\mathbf{X}}=\underset{\mathbf{S} \in \mathbb{S}}{\arg \min }\|\mathbf{Y}-\mathbf{H S}\|_{F}^{2},
$$

where $\mathbb{S}$ is the set of the proposed GSTBC-SM symbols.

\section{Performance Analysis for the Proposed GSTBC-SM SYSTEMS}

In this section, the orthogonality and ABEP of our proposed GSTBC-SM systems are analyzed.

\section{A. Orthogonality Analysis}

In this section, the orthogonality of the abovementioned STBC-SM schemes are analyzed.
1) Proposed $O S T B C-S M, R=0.75$ :

For the proposed OSTBC-SM scheme with $\mathrm{R}=0.75$, assuming that $\left(l_{1}, s_{1}\right),\left(l_{2}, s_{2}\right)$ and $\left(l_{3}, s_{3}\right)$ are the indices and M-APM symbols of $\mathbf{x}_{1}, \mathbf{x}_{2}$ and $\mathbf{x}_{3}$, we have

$$
\begin{aligned}
& \mathbf{S}_{434} \mathbf{S}_{434}^{H} \\
& =\left[\begin{array}{cccc}
\mathbf{x}_{1}-\mathbf{x}_{2}^{*} & \mathbf{x}_{3}^{*} & \mathbf{O} \\
\mathbf{x}_{2} & \mathbf{x}_{1}^{*} & \mathbf{O} & \mathbf{x}_{3}^{*} \\
\mathbf{x}_{3} & \mathbf{O} & -\mathbf{x}_{1}^{*} & -\mathbf{x}_{2}^{*} \\
\mathbf{O} & \mathbf{x}_{3} & \mathbf{x}_{2} & -\mathbf{x}_{1}
\end{array}\right]\left[\begin{array}{cccc}
\left(\mathbf{x}_{1}\right)^{H} & \left(\mathbf{x}_{2}\right)^{H} & \left(\mathbf{x}_{3}\right)^{H} & \mathbf{O} \\
\left(-\mathbf{x}_{2}^{*}\right)^{H} & \left(\mathbf{x}_{1}^{*}\right)^{H} & \mathbf{O} & \left(\mathbf{x}_{3}\right)^{H} \\
\left(\mathbf{x}_{3}^{*}\right)^{H} & \mathbf{O} & \left(-\mathbf{x}_{1}^{*}\right)^{H} & \left(\mathbf{x}_{2}\right)^{H} \\
\mathbf{O} & \left(\mathbf{x}_{3}^{*}\right)^{H} & \left(-\mathbf{x}_{2}^{*}\right)^{H} & \left(-\mathbf{x}_{1}\right)^{H}
\end{array}\right] \\
& {\left[\mathbf{O}_{N_{t} / 4}^{l_{1}, l_{1}^{*}}+\mathbf{O}_{N_{t} / 4}^{l_{2}^{*}, l_{2}}+\mathbf{O}_{N_{t} / 4}^{l_{3}^{*}, l_{3}} \quad \mathbf{O}_{N_{t} / 4}^{l_{1}, l_{2}^{*}}-\mathbf{O}_{N_{t} / 4}^{l_{2}^{*}, l_{1}}\right.} \\
& =\left[\begin{array}{cc}
\mathbf{O}_{N_{t} / 4}^{l_{2}, l_{1}^{*}}-\mathbf{O}_{N_{t} / 4}^{l_{1}^{*}, l_{2}} & \mathbf{O}_{N_{t} / 4}^{l_{1}, l_{1}^{*}}+\mathbf{O}_{N_{t} / 4}^{l_{2}^{*}, l_{2}}+\mathbf{O}_{N_{t} / 4}^{l_{3}^{*}, l_{3}} \\
\mathbf{O}_{N_{t} / 4}^{l_{3}, l_{1}^{*}}-\mathbf{O}_{N_{t} / 4}^{l_{1}^{*}, l_{3}} & \mathbf{O}_{N_{t} / 4}^{l_{3}, l_{2}^{*}}-\mathbf{O}_{N_{t} / 4}^{l_{2}^{*}, l_{3}} \\
\mathbf{O}_{N_{t} / 4}^{l_{2}, l_{3}}-\mathbf{O}_{N_{t} / 4}^{l_{3}, l_{2}} & \mathbf{O}_{N_{t} / 4}^{l_{3}, l_{1}}-\mathbf{O}_{N_{t} / 4}^{l_{1}, l_{3}}
\end{array}\right. \\
& \mathbf{O}_{N_{t} / 4}^{l_{1}, l_{3}^{*}}-\mathbf{O}_{N_{t} / 4}^{l_{3}^{*}, l_{1}} \\
& \left.\begin{array}{cc}
\mathbf{O}_{N_{t} / 4}^{l_{2}, l_{3}^{*}}-\mathbf{O}_{N_{t} / 4}^{l_{3}^{*}, l_{2}} & \mathbf{O}_{N_{t} / 4}^{l_{1}^{*}, l_{3}^{*}}-\mathbf{O}_{N_{t} / 4}^{l_{3}^{*}, l_{1}^{*}} \\
\mathbf{O}_{N_{t} / 4}^{l_{1}, l_{1}^{*}}+\mathbf{O}_{N_{t} / 4}^{l_{2}^{*}, l_{2}}+\mathbf{O}_{N_{t} / 4}^{l_{3}^{*}, l_{3}} & \mathbf{O}_{N_{t} / 4}^{l_{2}^{*}, l_{1}^{*}}-\mathbf{O}_{N_{t} / 4}^{l_{1}^{*}, l_{2}^{*}} \\
\mathbf{O}_{N_{t} / 4}^{l_{1}, l_{2}}-\mathbf{O}_{N_{t} / 4}^{l_{2}, l_{1}} & \mathbf{O}_{N_{t} / 4}^{l_{1}, l_{1}^{*}}+\mathbf{O}_{N_{t} / 4}^{l_{2}^{*}, l_{2}}+\mathbf{O}_{N_{t} / 4}^{l_{3}^{*}, l_{3}}
\end{array}\right],
\end{aligned}
$$

where we have

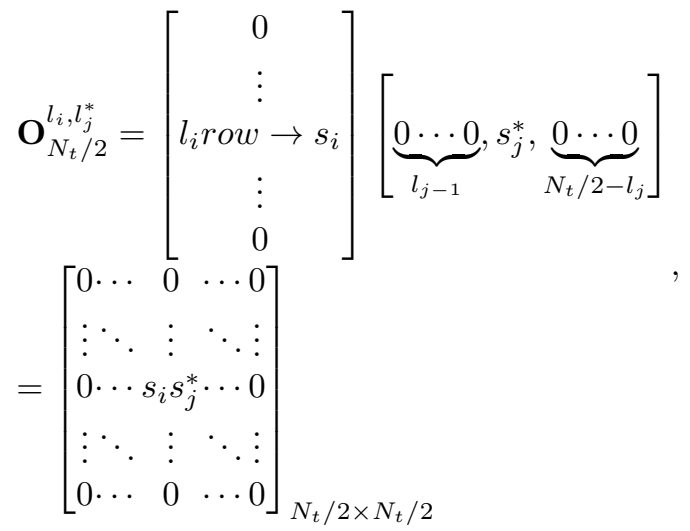

with

$$
\left\{\begin{array}{l}
\mathbf{O}_{N_{t} / 2}^{l_{i}, l_{j}^{*}}=\mathbf{O}_{N_{t} / 2}^{l_{j}^{*}, l_{i}}, \text { if } l_{i}=l_{j} \\
\mathbf{O}_{N_{t} / 2}^{l_{i}, l_{j}^{*}} \neq \mathbf{O}_{N_{t} / 2}^{l_{j}^{*}, l_{i}}, \text { if } l_{i} \neq l_{j}
\end{array}\right.
$$

2) Proposed $O S T B C-S M, R=0.5$ : For the proposed OSTBC-SM scheme with $\mathrm{R}=0.5$, assuming that $\left(l_{1}, s_{1}\right)$, $\left(l_{2}, s_{2}\right),\left(l_{3}, s_{3}\right)$ and $\left(l_{4}, s_{4}\right)$ are the indices and M-APM symbols of $\mathbf{x}_{1}, \mathbf{x}_{2}, \mathbf{x}_{3}$ and $\mathbf{x}_{4}$, the orthogonality analysis is presented in (15) of the top of the next page, where we have

$$
\begin{aligned}
& \Lambda_{448}=\mathbf{O}_{N_{t} / 4}^{l_{1}, l_{1}^{*}}+\mathbf{O}_{N_{t} / 4}^{l_{2}, l_{2}^{*}}+\mathbf{O}_{N_{t} / 4}^{l_{3}, l_{3}^{*}}+\mathbf{O}_{N_{t} / 4}^{l_{4}, l_{4}^{*}}, \\
& \Delta_{N_{t} / 4}^{i, j}=\mathbf{O}_{N_{t} / 4}^{l_{i}, l_{j}^{*}}-\mathbf{O}_{N_{t} / 4}^{l_{i}^{*}, l_{j}}, \\
& 2 \Re\left(\Delta_{N_{t} / 4}^{i, j}=\Delta_{N_{t} / 4}^{i, j}+\left(\Delta_{N_{t} / 4}^{i, j}\right)^{*} .\right.
\end{aligned}
$$

It is shown in (12) and (15) that the orthogonality of our proposed OSTBC-SM schemes are well.

\section{B. ABEP Analysis of $G S T B C-S M$}

We denote the transmit and receive signal of GSTBC$\mathrm{SM}$ by $\mathbf{S}_{i}$ and $\mathbf{S}_{j}$, respectively. Then the ABEP upper 


$$
\begin{aligned}
& \mathbf{S}_{448} \mathbf{S}_{448}^{H}=\left[\begin{array}{cccccccc}
\mathbf{x}_{1}-\mathbf{x}_{2} & -\mathbf{x}_{3} & -\mathbf{x}_{4} \mathbf{x}_{1}^{*}-\mathbf{x}_{2}^{*} & -\mathbf{x}_{3}^{*} & -\mathbf{x}_{4}^{*} \\
\mathbf{x}_{2} & \mathbf{x}_{1} & \mathbf{x}_{4} & -\mathbf{x}_{3} \mathbf{x}_{2}^{*} & \mathbf{x}_{1}^{*} & \mathbf{x}_{4}^{*} & -\mathbf{x}_{3}^{*} \\
\mathbf{x}_{3}-\mathbf{x}_{4} & \mathbf{x}_{1} & \mathbf{x}_{2} & \mathbf{x}_{3}^{*} & -\mathbf{x}_{4}^{*} & \mathbf{x}_{1}^{*} & \mathbf{x}_{2}^{*} \\
\mathbf{x}_{4} & \mathbf{x}_{3} & -\mathbf{x}_{2} & \mathbf{x}_{1} & \mathbf{x}_{4}^{*} & \mathbf{x}_{3}^{*} & -\mathbf{x}_{2}^{*} & \mathbf{x}_{1}^{*}
\end{array}\right] \times\left[\begin{array}{cccc}
\left(\mathbf{x}_{1}\right)^{H} & \left(\mathbf{x}_{2}\right)^{H} & \left(\mathbf{x}_{3}\right)^{H} & \left(\mathbf{x}_{4}\right)^{H} \\
-\left(\mathbf{x}_{2}\right)^{H} & \left(\mathbf{x}_{1}\right)^{H} & -\left(\mathbf{x}_{4}\right)^{H} & \left(\mathbf{x}_{3}\right)^{H} \\
-\left(\mathbf{x}_{3}\right)^{H} & \left(\mathbf{x}_{4}\right)^{H} & \left(\mathbf{x}_{1}\right)^{H} & -\left(\mathbf{x}_{2}\right)^{H} \\
-\left(\mathbf{x}_{4}\right)^{H} & -\left(\mathbf{x}_{3}\right)^{H} & \left(\mathbf{x}_{2}\right)^{H} & \left(\mathbf{x}_{1}\right)^{H} \\
\left(\mathbf{x}_{1}^{*}\right)^{H} & \left(\mathbf{x}_{2}^{*}\right)^{H} & \left(\mathbf{x}_{3}^{*}\right)^{H} & \left(\mathbf{x}_{4}^{*}\right)^{H} \\
-\left(\mathbf{x}_{2}^{*}\right)^{H} & \left(\mathbf{x}_{1}^{*}\right)^{H} & -\left(\mathbf{x}_{4}^{*}\right)^{H} & \left(\mathbf{x}_{3}^{*}\right)^{H} \\
-\left(\mathbf{x}_{3}^{*}\right)^{H} & \left(\mathbf{x}_{4}^{*}\right)^{H} & \left(\mathbf{x}_{1}^{*}\right)^{H} & -\left(\mathbf{x}_{2}^{*}\right)^{H} \\
-\left(\mathbf{x}_{4}^{*}\right)^{H} & -\left(\mathbf{x}_{3}^{*}\right)^{H} & \left(\mathbf{x}_{2}^{*}\right)^{H} & \left(\mathbf{x}_{1}^{*}\right)^{H}
\end{array}\right]
\end{aligned}
$$

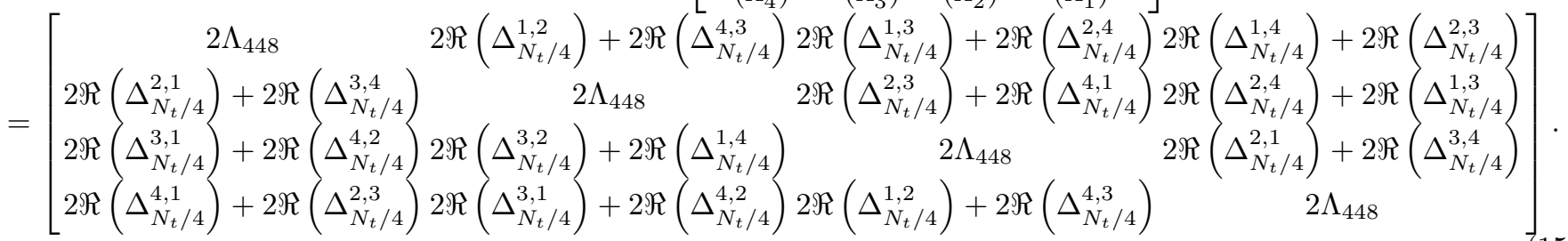

$$
\begin{aligned}
& P\left(\mathbf{S}_{i} \rightarrow \mathbf{S}_{j}\right)=\frac{1}{\pi} \int_{0}^{\pi / 2}\left(\frac{1}{1+\frac{\lambda_{i, j, 1}}{4 \sigma^{2} \sin ^{2} \phi}}\right)^{N_{r}} \times\left(\frac{1}{1+\frac{\lambda_{i, j, 2}}{4 \sigma^{2} \sin ^{2} \phi}}\right)^{N_{r}} \times \cdots \times\left(\frac{1}{1+\frac{\lambda_{i, j, \kappa_{i, j}}}{4 \sigma^{2} \sin ^{2} \phi}}\right)^{N_{r}} d \phi
\end{aligned}
$$

bound is given by

$$
P_{b}=\frac{1}{2 B 2^{2 B}} \sum_{i=1}^{2^{2 B}} \sum_{j=1, j \neq i}^{2^{2 B}} d\left(\mathbf{S}_{i}, \mathbf{S}_{j}\right) P\left(\mathbf{S}_{i} \rightarrow \mathbf{S}_{j}\right),
$$

where $d\left(\mathbf{S}_{i}, \mathbf{S}_{j}\right)$ is the number of error bits and $P\left(\mathbf{S}_{i} \rightarrow\right.$ $\mathbf{S}_{j}$ ) denotes the Pairwise Error Probability (PEP). According to [23], the value of $P\left(\mathbf{S}_{i} \rightarrow \mathbf{S}_{j}\right)$ can be obtained by Eq. (17), where $\kappa_{i, j}$ is the rank of the distance matrix $\mathbf{D}_{i, j}=\left(\mathbf{S}_{i}-\mathbf{S}_{j}\right)\left(\mathbf{S}_{i}-\mathbf{S}_{j}\right)^{H}$, and $\lambda_{i, j, 1}, \cdots, \lambda_{i, j, \kappa_{i, j}}$ are the non-zero eigenvalues of $\mathbf{D}_{i, j}$.

\section{Simulation Results}

In this section, we compare the performances of the proposed GSTBC-SM systems with conventional GSTBC systems. In all the simulation results, perfect channel state information is assumed and ML detector is employed. Moreover, the theoretical performances of both the proposed GSTBC-SM system and conventional STBC schemes are added for benchmarkers. As seen from Figs. 2-6, the upper bound derived becomes very tight upon increasing the SNR values for all the systems.

Specifically, Fig. 2 compares the performance of the proposed GSTBC-SM system using orthogonal coding with $R=0.5$ to that of conventional OSTBC with $R=0.5$ at 1.5 bpc. $\left(N_{t}, N_{u}, N_{r}, M\right)=(8,4,1,4)$ and $\left(N_{t}, N_{u}, N_{r}, M\right)=(8,4,2,4)$ are employed for our proposed GSTBC-SM scheme, while $\left(N_{t}, N_{u}, N_{r}, M\right)=$ $(4,4,1,8)$ and $\left(N_{t}, N_{u}, N_{r}, M\right)=(4,4,2,8)$ are employed for conventional STBC scheme. It is evident that the proposed GSTBC-SM scheme with $(8,4,1,4)$ is capable of outperforming conventional STBC scheme with $(4,4,1,8)$ by $5 \mathrm{~dB}$ at $\mathrm{BER}=10^{-5}$. The similar trend can be observed for the case of $N_{r}=2$.
Fig. 3 compares the performance of the proposed GSTBC-SM system using orthogonal coding with $R=0.5$ to that of conventional OSTBC with $R=0.5$ at $2 \mathrm{bpc}$. $\left(N_{t}, N_{u}, N_{r}, M\right)=(16,4,1,4)$ and $\left(N_{t}, N_{u}, N_{r}, M\right)=$ $(16,4,2,4)$ are employed for our proposed GSTBCSM scheme, while $\left(N_{t}, N_{u}, N_{r}, M\right)=(4,4,1,16)$ and $\left(N_{t}, N_{u}, N_{r}, M\right)=(4,4,2,16)$ are employed for conventional STBC scheme. It is evident that the proposed GSTBC-SM scheme is capable of outperforming conventional STBC scheme by around $10 \mathrm{~dB}$ at $\mathrm{BER}=10^{-5}$ for both the cases of $N_{r}=1, N_{r}=2$.

Fig. 4 compares the performance of the proposed GSTBC-SM system using orthogonal coding with $R=$ 0.75 to that of conventional corresponding OSTBC with $R=0.75$ at 2.25 bpc. $\left(N_{t}, N_{u}, N_{r}, M\right)=(8,3,1,4)$ and $\left(N_{t}, N_{u}, N_{r}, M\right)=(8,3,2,4)$ are employed for our proposed GSTBC-SM scheme, while $\left(N_{t}, N_{u}, N_{r}, M\right)=$ $(4,3,1,8)$ and $\left(N_{t}, N_{u}, N_{r}, M\right)=(4,3,2,8)$ are employed for conventional STBC scheme. It is evident that the proposed GSTBC-SM scheme with is capable of outperforming conventional OSTBC scheme with by around 4 $\mathrm{dB}$ at $\mathrm{BER}=10^{-5}$ for both the cases of $N_{r}=1, N_{r}=2$.

Fig. 5 compares the performance of the proposed GSTBC-SM system using orthogonal coding with $R=$ 0.75 to that of conventional corresponding OSTBC with $R=0.75$ at 3 bpc. $\left(N_{t}, N_{u}, N_{r}, M\right)=(16,3,1,4)$ and $\left(N_{t}, N_{u}, N_{r}, M\right)=(16,3,2,4)$ are employed for our proposed GSTBC-SM scheme, while $\left(N_{t}, N_{u}, N_{r}, M\right)=$ $(4,3,1,16)$ and $\left(N_{t}, N_{u}, N_{r}, M\right)=(4,3,2,16)$ are employed for conventional STBC scheme. It is evident that the proposed GSTBC-SM scheme with $(16,3,1,4)$ is capable of outperforming conventional OSTBC scheme with $(4,3,1,16)$ by around $8 \mathrm{~dB}$ at $\mathrm{BER}=10^{-5}$. The performance advantages will become more dominant for the case of $N_{r}=2$. 


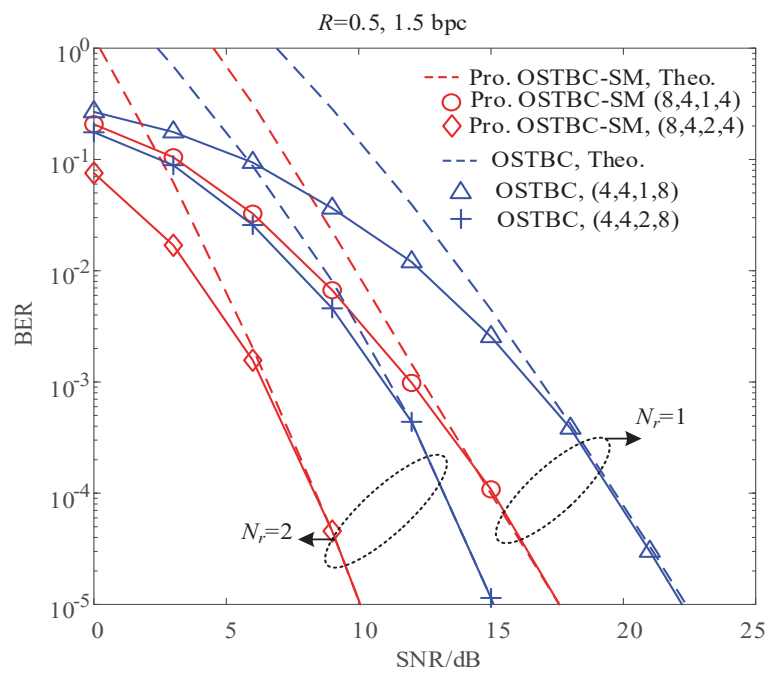

Fig. 2. Performance comparison between the proposed GSTBC-SM and the conventional OSTBC schemes at $1.5 \mathrm{bpcu}$.

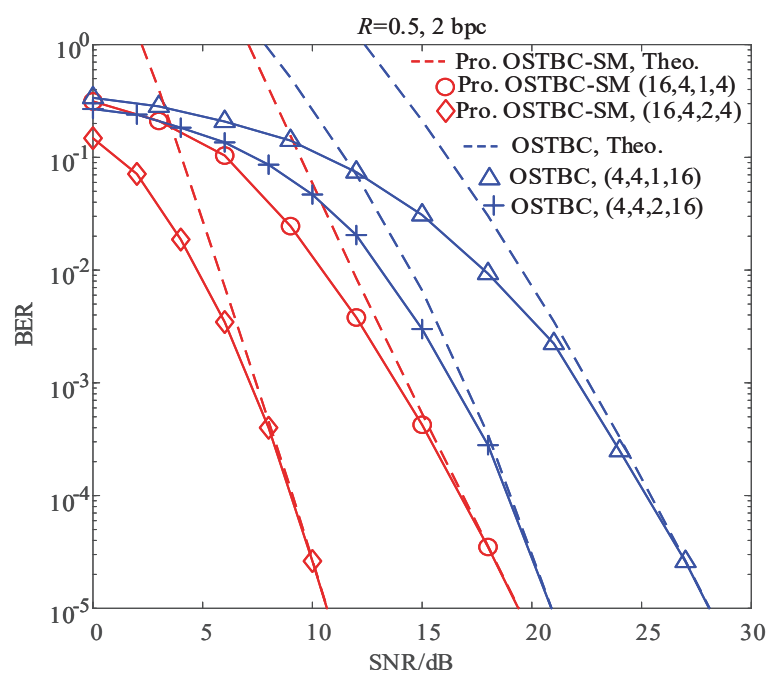

Fig. 3. Performance comparison between the proposed GSTBC-SM and the conventional OSTBC schemes at $2 \mathrm{bpcu}$.

In order to provide further insights, Fig. 6 compares the performance of the proposed GSTBC-SM system using orthogonal coding with $R=0.75$ to that of existing Alamouti-SM of [24]] at $3 \mathrm{bpc} .\left(N_{t}, N_{u}, N_{r}, M\right)=$ $(8,3,1,8)$ and $\left(N_{t}, N_{u}, N_{r}, M\right)=(8,3,2,8)$ are employed for our proposed GSTBC-SM scheme, while $\left(N_{t}, N_{u}, N_{r}, M\right)=(8,2,1,2)$ and $\left(N_{t}, N_{u}, N_{r}, M\right)=$ $(8,2,2,2)$ are employed for Alamouti-SM of [24]. It is evident that the proposed GSTBC-SM scheme is capable of outperforming Alamouti-SM by around $10 \mathrm{~dB}$ at $\mathrm{BER}=10^{-5}$ for the case of $N_{r}=1$. As $N_{r}$ increases, the diversity gain will become slight.

\section{CONCLUSIONS}

In this paper, a generalized STBC based SM system is proposed, which incorporates the $\mathrm{SM}$ concept into

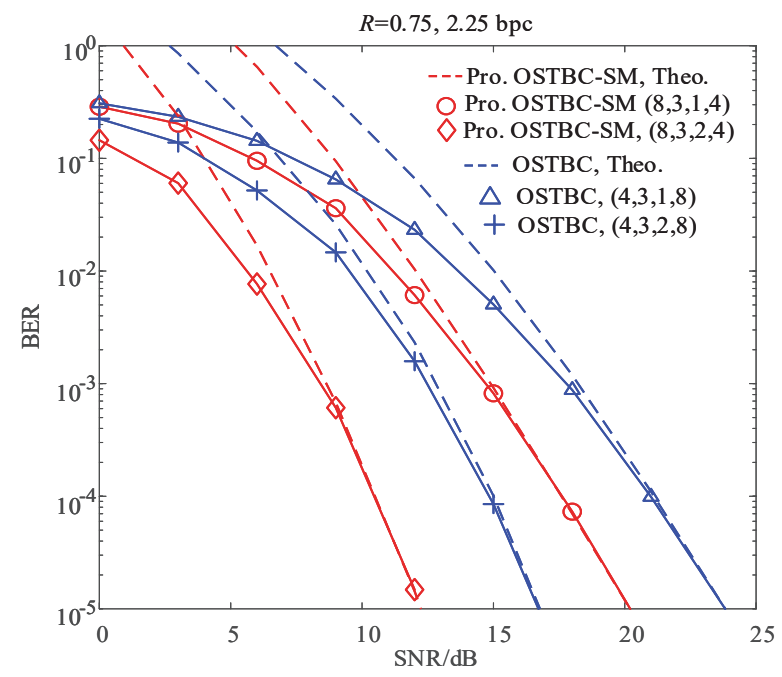

Fig. 4. Performance comparison between the proposed GSTBC-SM and the conventional OSTBC schemes at $2.25 \mathrm{bpcu}$.

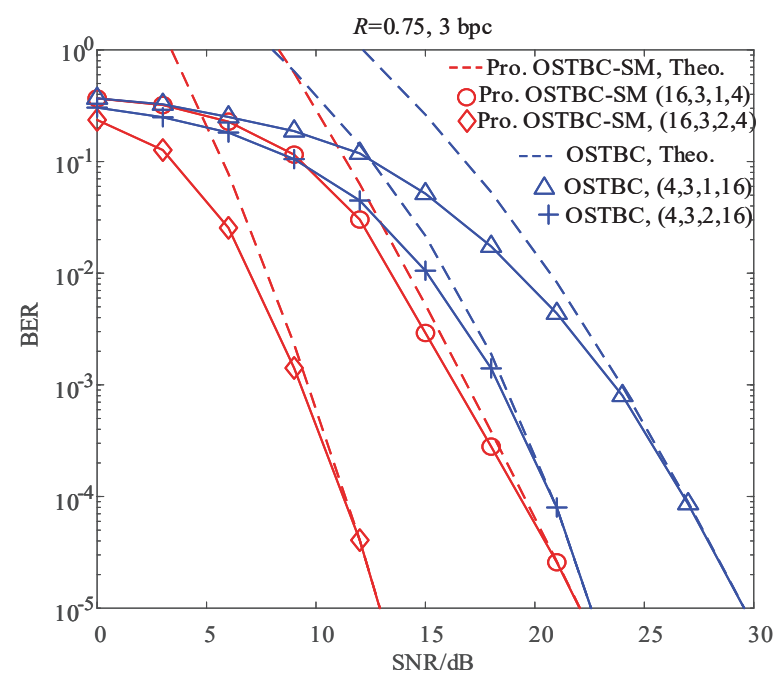

Fig. 5. Performance comparison between the proposed GSTBC-SM and the conventional OSTBC schemes at $3 \mathrm{bpcu}$.

the classic STBC structure to further exploit the diversity gain. Both simulation and theoretical results have shown that the proposed GSTBC-SM system is capable of providing considerable performance gains over the corresponding STBC schemes. Moreover, the proposed OSTBC-SM scheme with $R=0.75$ ratio is capable of providing significant performance gain over the existing Alamouti-SM scheme.

\section{ACKNOWLEDGEMENT}

This work was supported in part by the U.K. Engineering and Physical Sciences Research Council under Grant EP/N020391/1. The authors also would like to acknowledge the support of the University of Surrey 5GIC (http://www.surrey.ac.uk/5gic) members for this work. 


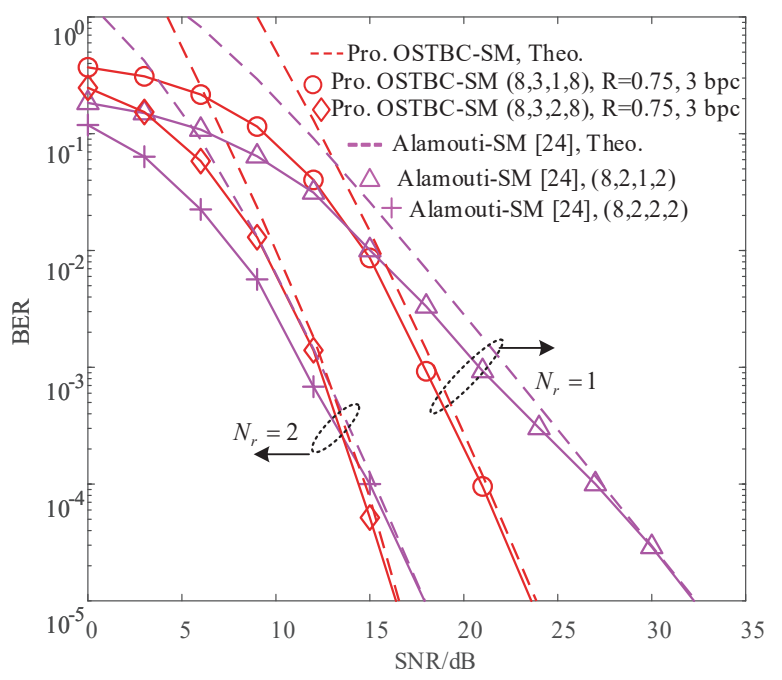

Fig. 6. Performance comparison between the proposed GSTBC-SM and the existing Alamouti-SM schemes at $3 \mathrm{bpcu}$.

\section{REFERENCES}

[1] S. Alamouti. "A simple transmit diversity technique for wireless communications," IEEE Journal on Selected Areas in Communications, vol.16, no.8, pp. 1451-1458, Oct. 1998.

[2] V. Tarokh, N. Seshadri, and A. R. Calderbank, "Space-time codes for high data rate wireless communication: Performance criterion and code construction," IEEE Transcations on Information Theory, vol. 44, no. 2, pp. 744-765, Mar. 1998.

[3] V. Tarokh, H. Jafarkhani, and A. R. Calderbank, "Space-time block codes from orthogonal designs," IEEE Transcations on Information Theory, 1999, vol. 45, no. 5, pp. 1456-1467, July, 1999.

[4] W. Su, X. Xia, and K. J. Liu, "A Systematic Design of HighRate Complex Orthogonal Space-Time Block Codes,", IEEE Commun. Lett., vol. 8, no. 6, June 2004. 380-382,

[5] H. Jafarkhani. "A quasi-orthogonal space-time block code," IEEE Transactions on Communications, vol. 49, no. 1, pp. 1-4, Jan. 2001.

[6] W. Su, X. Xia, "Signal constellations for quasi-orthognal space time block codes with full diversity," IEEE Transcations on Information Theory, vol. 50, no. 10, pp. 2331-2347, Oct. 2004.

[7] L. A. Dalton and C. N. Georghiades, "A full-rate, full-diversity four-antenna quasi-orthogonal space-time block code," IEEE Transactions on Wireless Communications, vol. 4, no. 2, pp. 363-366, 2005.

[8] W. Song, M. Lee, M. Matalgah and Y. Guo. "Quasi-Orthogonal Space-Time Block Codes Designs Based on Jacket Transform," Jounral of Communication and Networks, vol. 12, no. 3, pp. 240-245, June, 2010,

[9] Z. Lei, C. Yuen and F. Chin, "Quasi-orthogonal space-time block codes for two transmit antennas and three time slots,"
IEEE Transactions on Wireless Communications, vol. 10, no. 6, pp. 1983-1991, June, 2011.

[10] L. Wang and Z. Chen. "Spatially modulated diagonal space time code," IEEE Commun. Lett., vol. 19, no. 7, pp. 1245-1248, July, 2015.

[11] V. Vakilian and H. Mehrpouyan, "High rate and low-complexity space-time block codes for $2 \times 2$ MIMO systems," IEEE Commun. Lett., vol. 20, no. 6, 1227-1230, June, 2016.

[12] R. Mesleh, H. Haas, S. Sinanovic, C. W. Ahn, and S. Yun, "Spatial modulation," IEEE Trans. Veh. Technol., vol. 57, no. 4, pp. 2228-2241, Jul. 2008.

[13] M. Di Renzo, H. Haas, and P. M. Grant, "Spatial modulation for multiple-antenna wireless systems: A survey," IEEE Commun. Mag., vol. 49, no. 12, pp. 182-191, Dec. 2011.

[14] M. Di Renzo, H. Haas, A. Ghrayeb, S. Sugiura, and L. Hanzo, "Spatial modulation for generalized MIMO: challenges, opportunities and implementation," Proceedings of the IEEE, vol. 102, no. 1, pp. 56-103, Jan. 2014

[15] P. Yang, M. Di Renzo, Y. Xiao, S. Q. Li and L. Hanzo, "Design guidelines for spatial modulation," IEEE Commun. Surveys Tuts., vol. 17, no. 1, pp. 6-26, First Quart. 2015.

[16] P. Yang, Y. Xiao, Y. L. Guan, K. V. S. Hari, A. Chockalingam, S. Sugiura, H. Haas, M. Di Renzo, C. Masouros, Z. Liu, L. Xiao, S. Li, and L. Hanzo, "Single-carrier spatial modulation: A promising design for large-scale broadband antenna systems," IEEE Commun. Surveys Tuts., vol. 18, no. 3, pp. 1687-1716, Feb. 2016.

[17] E. Basar, "Index modulation techniques for 5G wireless networks," IEEE Commun. vol. 54, no. 7, pp. 168-175. 2016.

[18] E. Basar, M. Wen, R. Mesleh, M. D. Renzo, Y. Xiao and H. Haas, "Index modulation techniques for next-generation wireless networks," IEEE Access, pp. 16693-16746, Aug. 2017.

[19] E. Basar, U. Aygolu, E. Panayirci and H. V. Poor, "Space-Time Block Coded Spatial Modulation," IEEE Trans. Commun., vol. 59, no. 3, pp. 823-832, Mar. 2011.

[20] X. Li and L. Wang, "High rate space-time block coded spatial modulation with cyclic structure,"IEEE Commun. Lett., vol. 18, no. 4, pp. 532-535, Apr. 2014.

[21] C. Jeon and J. Lee, "Multi-strata space-time coded spatial modulation," IEEE Commun. Lett., vol. 19, no. 11, pp. 19451948, Nov. 2015.

[22] Y. Hua, G. Zhao, W. Zhao, and M. Jin, "Modified codewords design for spaceĺCtime block coded spatial modulation," IEEE Trans. IET Commun., vol. 11, no. 2, pp. 249-257, Jan. 2017.

[23] M. C. Park, B. G. Jo and D. S. Han, "Double space-time transmit diversity with spatial modulation," IEEE Electronic Lett., vol. 51, no. 25, pp. 2155-2156, Dec. 2015.

[24] L. Xiao, Y. Xiao, L. You, P. Yang, S. Li and L. Hanzo, "SingleRF and Twin-RF spatial modulation for an arbitrary number of transmit antennas," IEEE Trans. Veh. Tech., vol. pp, no. 12, Dec. 2017.

[25] M. Le, V. Ngo, H. Mai, X. Nam, and M. Di. Renzo, "Spatially modulated orthogonal space-time block codes with nonvanishing determinants," IEEE Trans. Commun., vol. 62, no. 1, pp. 85-99, Jan. 2014. 